\title{
Bronchial gland duct ectasia in fatal bronchial asthma: association with interstitial emphysema
}

\author{
A Cluroe, L HOLloway, K THOMSON, G PURDie, $\uparrow$ R BEASLEY* \\ From the Departments of Pathology, ${ }^{*}$ Medicine, and $\nmid$ Community Health, Wellington School of Medicine, \\ Wellington, New Zealand
}

SUMMARY To determine the incidence of bronchial gland duct ectasia in fatal asthma and its association with interstitial emphysema, the histological features of 72 patients in whom death was considered to be due to asthma, and 72 matched control subjects in whom sudden death was not attributed to asthma, were reviewed. In all cases and controls, sections of two or more blocks of lung tissue stained with haematoxylin and eosin were obtained at necropsy. Bronchial gland duct ectasia was diagnosed if there was more than one abnormally dilated epithelial lined protrusion from a bronchus, extending through the smooth muscle layer. A histological diagnosis of asthma was made if four of the five following criteria were present: mucus plugging, basement membrane thickening, epithelial shedding, submucosal eosinophil leucocyte infiltration and smooth muscle hypertrophy.

A histological diagnosis of asthma was made in 53 of 72 clinical cases of fatal asthma and in five of $\stackrel{\circ}{\circ}$ 72 control subjects. Interstitial emphysema was present in 10 clinical cases of fatal asthma, all of ${ }_{\mathbb{\Phi}}$ whom had bronchial gland duct ectasia and a histological diagnosis of asthma. Interstitial emphysema was not observed in control subjects.

It is concluded that bronchial gland duct ectasia is a common histological feature of severe asthmm and that interstitial emphysema may be consequent on rupture of these dilated gland ducts.

The histological features which are considered to be characteristic of fatal asthma include mucus plugging, denudation of the respiratory epithelium, basement membrane thickening, goblet cell metaplasia, smooth muscle hyperplasia and eosinophil cell infiltration..$^{1-5}$ The presence of dilated seromucinous gland ducts arising from the bronchi, however, is one feature that has received little attention by pathologists since it was first described by Monckeberg in 1909.6 In his description of a case of fatal asthma bronchi of 0.4 to $0.5 \mathrm{~mm}$ diameter were observed to have diverticula or cryptlike protrusions of epithelium (Schleimhaustausstülpungen) that interrupted the muscle layer. These findings were confirmed in 1922 by Huber and Koessler, who noted that numerous large gland-duct ampullae filled with mucus were present in bronchi in half of their cases of fatal asthma. ${ }^{1}$ Subsequently, these structures have been identified by several authors and referred to as bronchial sacculations, ${ }^{7}$ diverticulosis of the epithelium, ${ }^{2}$ dilatation of gland openings, ${ }^{8}$ and bronchial diverticula. ${ }^{9}$ In these descriptions the dilated structures have been shown to have an intact basement membrane and to extend through the musculature. It

Accepted for publication 25 May 1989 has been suggested that they develop as a result of raised intrabronchial tension and that they have their origin at the mouth of mucus glands. Although the morphology and possible pathogenesis of these ectatic bronchial gland ducts have been well documented, their clinical importance is uncertain.

More recently we showed the presence of a ruptured dilated duct in histological sections from a subject in whom a fatal asthma attack was complicated by severe interstitial emphysema. ${ }^{10}$ As a result of these observations, we reviewed a large series of asthma deaths to confirm the histological features of bronchial gland duct ectasia, their incidence in fatal asthma, and to determine their association with other histological features of asthma, in particular interstitial emphysema.

\section{Material and methods}

Eighty two cases of death due to bronchial asthma were identified from the records of Wellington District Coroner's Pathologists. This represented all cases arising over a period of 16 years (1971-1987) in which necropsy was performed and asthma diagnosed as the cause of death. These cases were matched by age and 


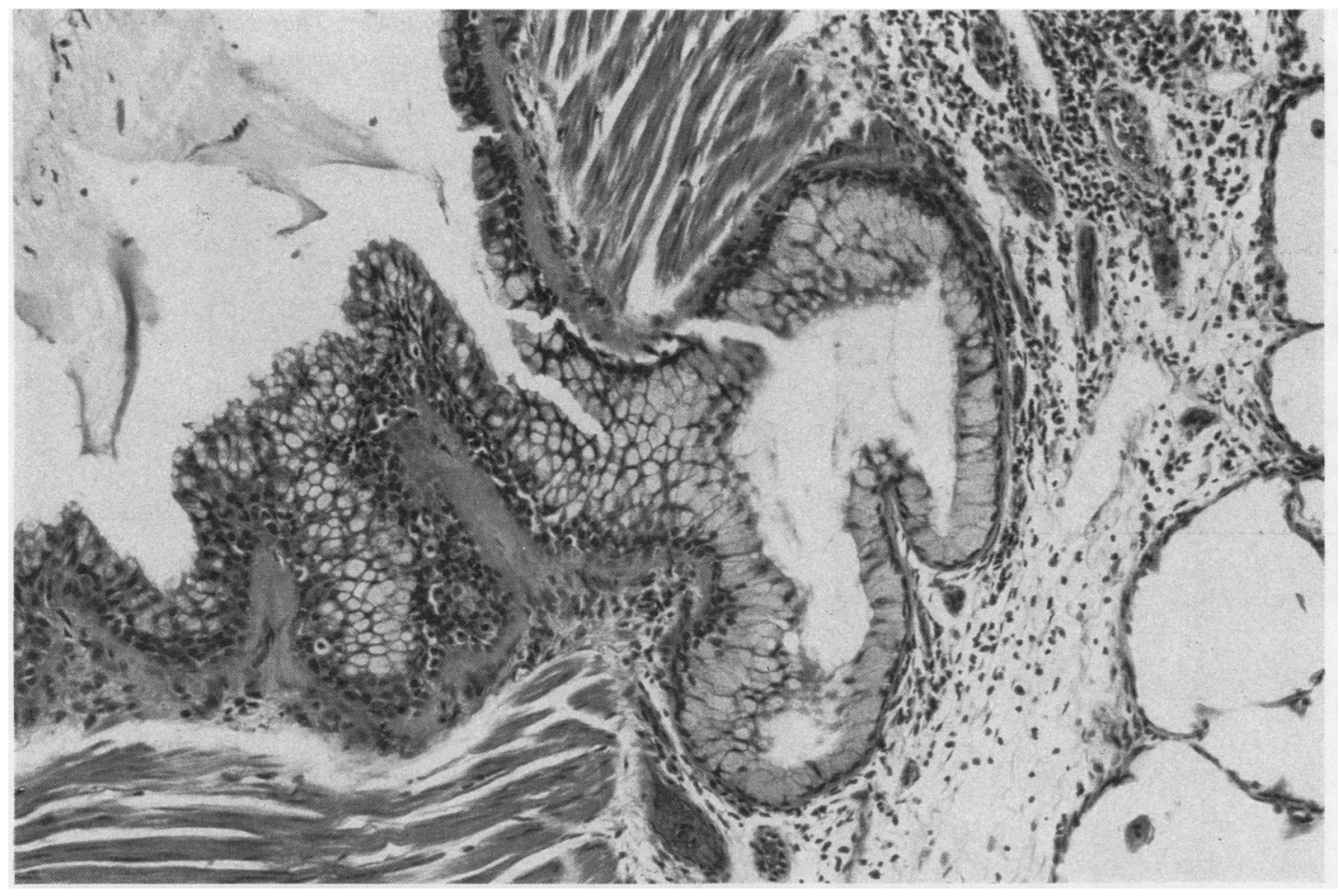

Fig 1 Dilated gland duct, lined by goblet cells, extending through the muscular wall of the bronchus. There is also basement membrane thickening and a mucus plug within the bronchial lumen.

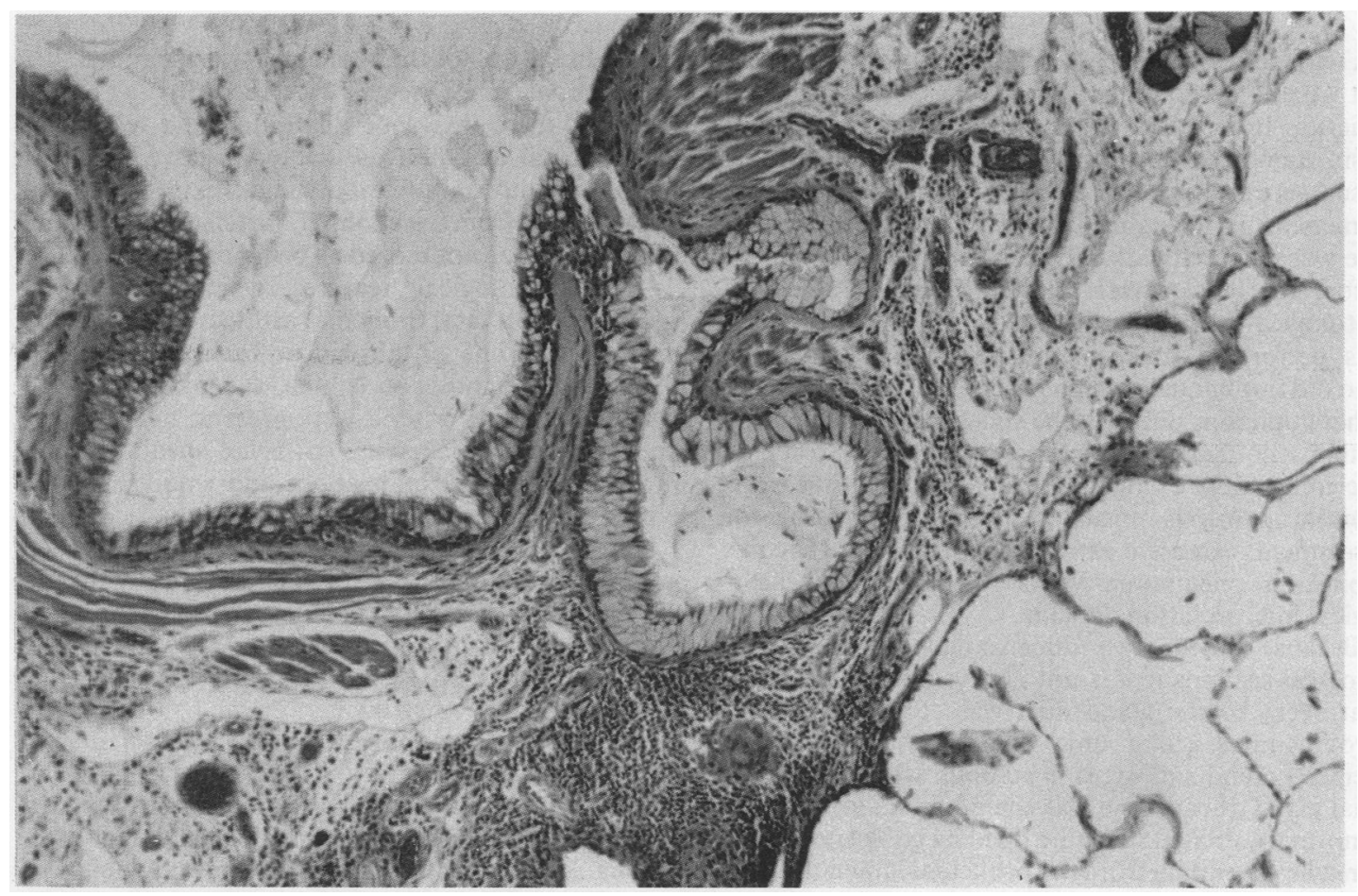

Fig 2 Bronchus showing a dilated duct with periductal inflammation. 


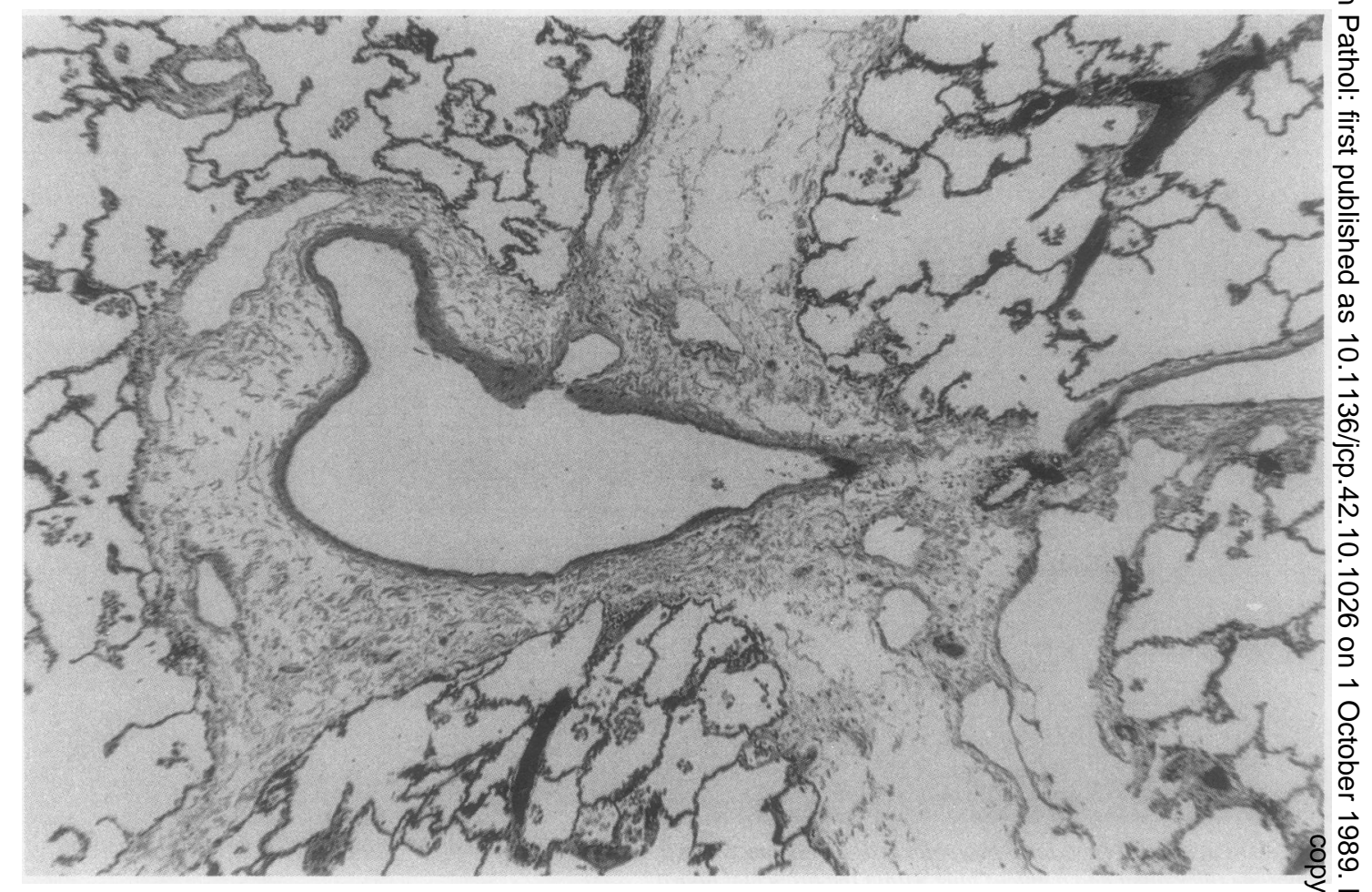

Fig 3 Interstitial emphysema with disruption and tearing of connective tissue planes.

sex with control subjects selected from sudden deaths not attributed to asthma from the same coroner's practice. In all cases and controls haematoxylin and eosin stained sections of two or more blocks of lung tissue were examined. Most tissue blocks were fixed by immersion in formalin. In a few cases the bronchial tree was perfused with formalin and left for 24 hours before the blocks were selected. The assessment of histological features in the study and control groups was performed independently by two pathologists who did not know to which group each case belonged. Where opinions differed, the slides were looked at by both pathologists jointly and a consensus reached. Specimens were excluded if there was extensive postmortem autolysis, insufficient bronchi present for assessment, extensive extra-bronchial disease such as aspiration pneumonia which would render histological interpretation difficult.

Because of the two dimensional nature of histological sections it was not always possible to show that every ectatic gland duct was connected with a seromucinous acinar unit, and many dilated ducts seemed to end simply as a bulbous structure. Bronchial gland duct ectasia was therefore diagnosed if an abnormally dilated structure lined by goblet or columnar cells, extending from the bronchial lumen through the muscular wall of the bronchus, was identified.

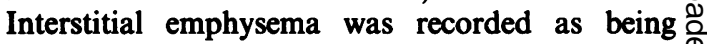
present if there was wide disruption and tearing of $\stackrel{\mathbb{Q}}{\Omega}$ peribronchial and perivascular connective tissue. If $\vec{\overrightarrow{ }}$ there was uncertainty in distinguishing interstitial 3 emphysema from sectioning artefact interstitial $\vec{F}$ emphysema was not scored as being present.

Other histological features which have been associated with fatal bronchial asthma were recorded ? as either present or absent in each specimen. $A \frac{5}{3}$ histological diagnosis of asthma was made if four of the five following criteria were present: mucus plug- $\frac{\mathrm{O}}{3}$ ging; basement thickening; epithelial shedding; submucosal eosinophil infiltration; and smooth muscle $\frac{\text { S }}{3}$ hypertrophy.

Differences in the presence of interstitial emphysema among cases of fatal asthma with and $N$ without ectatic bronchial gland ducts were compared by means of Fisher's exact test.

CASES OF FATAL ASTHMA

A histological diagnosis of asthma was made in 53 of 0 the 72 clinical cases of fatal asthma. Bronchial gland $\mathbb{\varnothing}$ duct ectasia was identified in specimens from 39 cases, $\stackrel{?}{-}$ all but three of whom had a histological diagnosis of ${ }^{0}$ asthma. Many of the dilated ducts showed goblet cell $\underset{\mathbb{\Phi}}{\stackrel{\circ}{\circ}}$ 


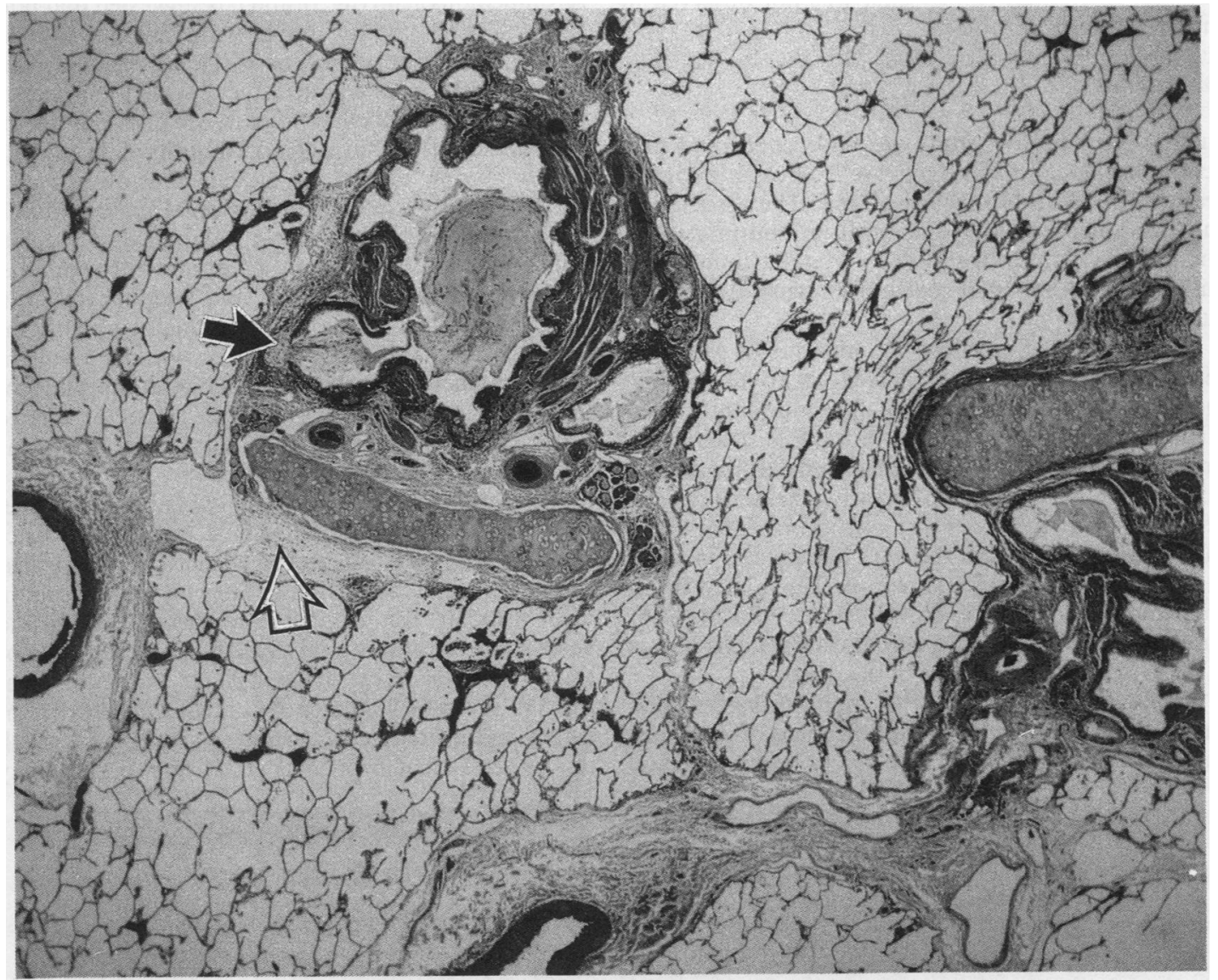

Fig 4 Low power photomicrograph of lung showing bronchi and pulmonary vessels. Note ruptured ectatic duct (closed arrow) and presence of interstitial emphysema (open arrow).

metaplasia around the necks while others were lined by either goblet cells (fig 1) or by ciliated and nonciliated columnar epithelium. In many instances, there was severe periductal inflammation (fig 2). This inflammatory component predominantly comprised

Table Association between bronchial gland duct ectasia and interstitial emphysema in 53 subjects with histological and clinical fatal asthma

\begin{tabular}{llcl}
\hline & \multicolumn{3}{l}{ Dilated gland ducts } \\
\cline { 2 - 4 } Interstitial emphysema & Present & Absent & Total \\
\hline Present & 10 & 0 & 10 \\
Absent & 26 & 17 & 43 \\
Total & 36 & 17 & 53 \\
\hline
\end{tabular}

$p=0.02$ Fisher's exact test. eosinophils, which in some cases seemed to infiltrate through the basement membrane to the epithelial surface of the dilated duct. The lumina of the dilated ducts were frequently distended with mucus.

Interstitial emphysema was identified in specimens from 10 cases, all of whom had bronchial gland duct ectasia and a histological diagnosis of asthma (table 1). Whenever interstitial emphysema was identified, there was clear disruption of tissue planes around bronchovascular bundles (fig 3). In one case interstitial emphysema was seen in association with a ruptured dilated duct (fig 4). There was a significant association between interstitial emphysema and bronchial gland duct ectasia $(p=0.02)$.

CONTROL SUBJECTS

A histological diagnosis of asthma was made in five of the 72 control subjects. Ectatic bronchial gland ducts were present in all five of these specimens, and in two 
cases who did not satisfy our histological criteria for asthma. Interstitial emphysema was not observed in any of the control subjects.

\section{Discussion}

In this retrospective study we have confirmed that ectatic bronchial gland ducts are a histopathological feature of severe bronchial asthma. The association that we noted between dilated ducts and interstitial emphysema would suggest that in fatal asthma interstitial emphysema may occur following rupture of such ducts.

Examination of necropsy specimens from this large series of asthma deaths allowed us to review the morphology and determine the incidence of bronchial gland duct ectasia in fatal asthma. Consistent with previous descriptions, ${ }^{1679}$ we observed that the dilated ducts extended through the muscular wall of the bronchus with the basement membrane intact, that there was mucus within the duct lumen, and in some instances periductal inflammation. The dilated bronchial gland ducts were lined with either goblet cells or columnar epithelium, and goblet cell metaplasia was often evident at the mouths of the ducts.

As previously proposed, the origin of these dilated structures is from the mouths of the ducts of peribronchial mucus glands. ${ }^{16-9}$ These ducts become ectatic proximal to their dividing into the terminal 0 seromucinous acini (fig 5), and it is suggested that both $\stackrel{*}{=}$ the airflow obstruction and airways inflammation are $\underline{0}$ important in their pathogenesis. Thus bronchial gland ducts may become ectatic after repeated episodes of $\stackrel{\overrightarrow{9}}{\rightarrow}$ increased intrabronchial pressure which occur during $\bar{c}$ episodes of asthma, or when the mouths of the mucous $\bar{c}$ glands become obstructed by tenacious intraluminal $\frac{\bar{\omega}}{\vec{D}}$ mucus, mucosal oedema, or bronchial smooth muscle $\stackrel{\mathbb{\Phi}}{\circ}$ contraction. There was often a pronounced periductal inflammatory cell infiltrate, predominantly comprising eosinophils, plasma cells, and lymphocytes. It is $?$ conceivable that release of inflammatory mediators $\vec{\omega}$ from these cells may contribute to weakening of the $\stackrel{\sigma}{\sigma}$ duct wall, thereby leading to duct dilatation. Thus the mechanisms leading to the development of dilated ducts in asthma are probably similar to those causing ? duct ectasia in chronic bronchitis. 112

We identified ectatic bronchial gland ducts in over $\vec{\circ}$ half of patients dying from asthma, with an incidence $N$ approaching that of features which are regarded as 은 characteristic of fatal asthma. They probably occur in most bronchi, for in most cases haematoxylin and $O$ eosin stained sections were available from only two or $\frac{?}{0}$ three blocks of tissue. Dilated ducts were also iden- ${ }_{\infty}$ tified in seven of 72 matched control subjects, five of whom had histological features of asthma. Although $\vec{\oplus}$ this suggests that bronchial gland duct ectasia mạ

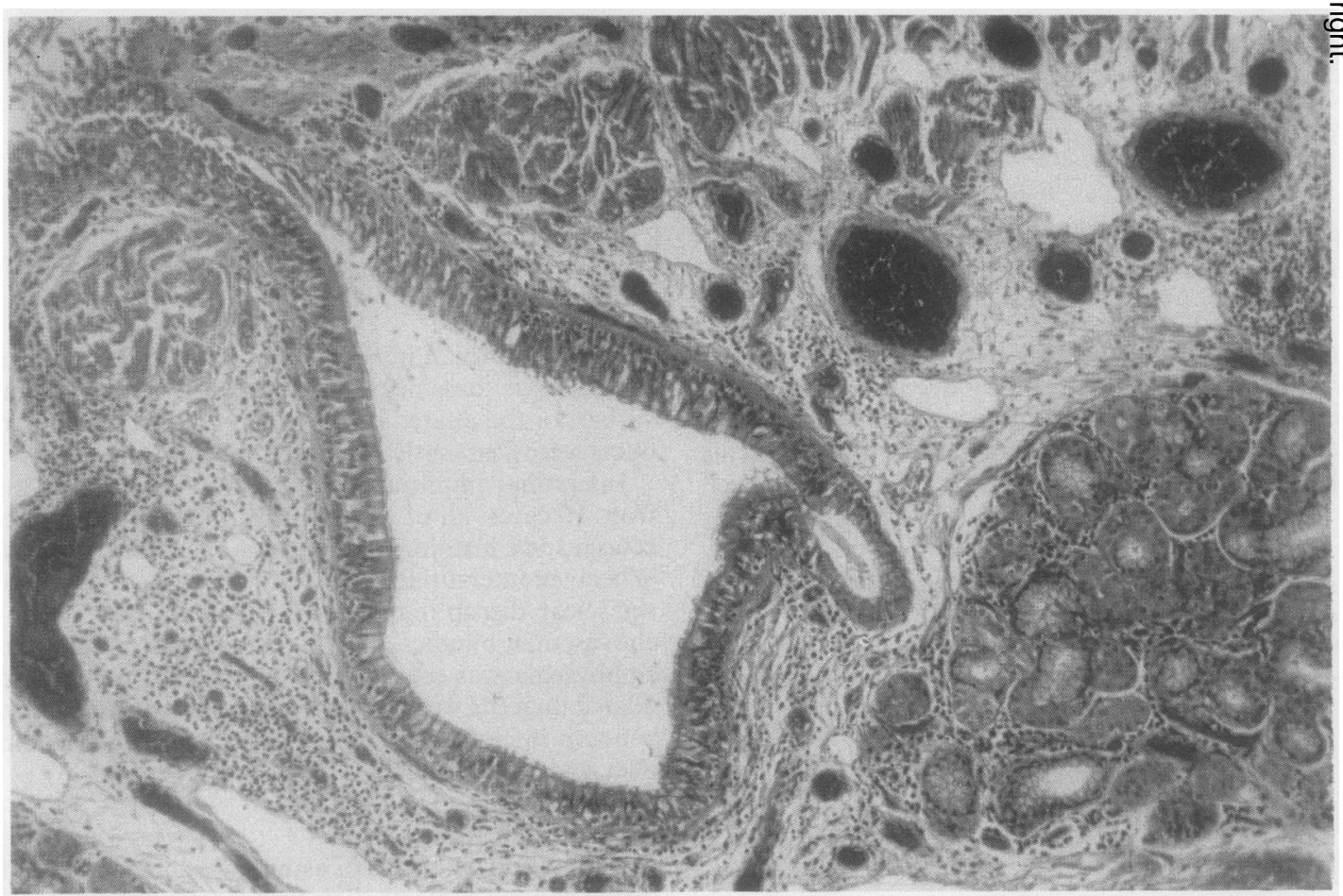

Fig 5 Ectatic bronchial gland duct lined by ciliated epithelium, showing its relation to terminal seromucinous acini. 
occur in non-fatal asthma, it is possible that some of these subjects may have died from an attack of asthma that was not recognised clinically. Alternatively, some of the control subjects may have had chronic bronchitis, in which ectatic bronchial glands have been observed to commonly occur. ${ }^{11} 12$

We were concerned that in cases of sudden death in known asthmatics a potential bias existed towards attributing asthma as the cause of death. For this reason we required that the clinical diagnosis was supported by definite histological evidence of asthma in the lung sections examined. If control subjects also had these characteristic pathological features, asthma was considered to be present, but not necessarily the cause of death. Because detailed clinical data were not available in most of the subjects, we used these conservative histological criteria to make a definitive diagnosis of asthma.

The pathogenesis of spontaneous interstitial emphysema in asthma was first proposed by Macklin and Macklin. ${ }^{1314}$ Although they suggested that the basic mechanism was the development of a pressure gradient across the alveolar wall sufficient to disrupt its integrity, the animal models used are not applicable to the clinical situation of asthma in which bronchial gland duct ectasia occur. Our findings support the hypothesis that interstitial emphysema complicating fatal asthma is not due to alveolar disruption, but rather to rupture of dilated bronchial gland ducts. Interstitial emphysema only occurred in asthmatic patients in whom dilated ducts were present, and was absent in matched control subjects in whom asthma was not the cause of death. Furthermore, in one of the fatal asthma cases, serial histological sections showed interstitial emphysema associated with definite rupture of an ectatic duct. As the pressure within the duct lumen increases from whatever cause, the duct also probably dilates. In accordance with Laplace's law $(\mathrm{T}=\mathrm{Pr} ; \mathrm{T}=$ tension in wall, $\mathrm{P}=$ intraluminal pressure, $r=$ radius of lumen) this dilatation would lead to a proportionate increase in the tension in the wall of the duct, increasing the chances of rupture.

Regardless of its site, when rupture occurs, air dissects along the perivascular and peribronchial spaces. The air may remain confined to the lung, or extend into the mediastinum and track to the subcutaneous tissues of the neck and chest. Although it is difficult to determine the contribution made by interstitial emphysema to the fatal outcome of an attack of asthma, its presence is likely to compromise further the cardiovascular and respiratory function of the affected patient. ${ }^{1516}$

The characteristic histological features of interstitial emphysema were observed in about $20 \%$ of our confirmed cases of fatal asthma. While this figure is greater than we would have expected, the absence of these histological features in our non-asthmatic subjects, and with the careful exclusion of changes due to shearing and sectioning artefact, we suggest that interstitial emphysema is a common complication of fatal asthma. This is consistent with the findings of Eggleston $e t$ al, who reported that $15 \%$ of children aged 10 years or older admitted with severe asthma had pneumomediastinum present on their admission radiographs. ${ }^{17}$

In conclusion, we suggest that bronchial gland duct ectasia is an important and underestimated histological feature of severe asthma, and that rupture of dilated ducts may be relevant to the pathogenesis of spontaneous interstitial emphysema, which may occur during and complicate an exacerbation of asthma.

We thank the Asthma Foundation of New Zealand and the Medical Research Council of New Zealand for financial support, and Mrs M Stretch and Mrs H Bark for typing the manuscript.

\section{References}

1 Huber HL, Koessler KK. The pathology of bronchial asthma. Arch Intern Med 1922;30:689-760.

2 Houston JC, de Navasquez S, Trounce JR. A clinical and pathological study of fatal cases of status asthmaticus. Thorax 1953;8:207-13.

3 Cardwell BS, Pearson RSB. Death in asthmatics. Thorax 1959;14:341-52.

4 Reid L. Pathological changes in asthma. In: Clark TJK, Godfrey S, eds. Asthma. London: Chapman and Hall, 1977:79-95.

5 Dunnill MS. The morphology of the airways in bronchial asthma. In: Stein M, ed. New directions in asthma. Park Ridge, Illinois: American College of Chest Physicians, 1975:213-21.

6 Mönckeberg JG. Zur Pathologischen Anatomie des Bronchialasthmas. Verhandl d deutsch pathol Gesellsch 1909;14:173-80.

7 Macdonald IG. The local and constitutional pathology of bronchial asthma. Ann Int Med 1933;6:253-77.

8 Lamson RW, Butt EM. Fatal "asthma". A clinical and pathologic consideration of 187 cases. JAMA 1937;108:1843-50.

9 Dunnill MS. The pathology of asthma, with special reference to changes in the bronchial mucosa. J Clin Pathol 1960;13:27-33.

10 Cluroe AD, Holloway L, Beasley R. Bronchial diverticulitis: a complication of bronchial asthma. J Clin Pathol 1988;41:921-2.

11 Simon G, Galbraith H-J B. Radiology of chronic bronchitis. Lancet 1953;ii:850-2.

12 Duprez A, Mampuys R. Cystic enlargement of the mucous glands of the bronchus associated with chronic bronchitis. Thorax 1953;8:141-7.

13 Macklin CC. Transport of air along sheaths of pulmonic blood vessels from alveoli to mediastinum. Clinical Implications. Arch Intern Med 1939;64:913-26.

14 Macklin MT, Macklin CC. Malignant interstitial emphysema of the lungs and mediastinum as an important complication of many respiratory diseases and other conditions. Interpretation of clinical literature in light of laboratory experiment. Medicine 1944;23:281-358.

15 Steffey WR, Cohn AM. Spontaneous subcutaneous emphysema of the head, neck and mediastinum. Arch Otolaryngol 1974;100:32-5.

16 D'Assumpcao C, Smith WG. Spontaneous mediastinal and subcutaneous emphysema complicating bronchial asthma. Med $J$ Aust 1967;1:328-30.

17 Eggleston PA, Ward BH, Pierson WE, Bierman CW. Radiographic abnormalities in acute asthma in children. Pediatrics 1974;54:442-9.

Requests for reprint requests to: Dr A Cluroe, Department of Pathology, Wellington School of Medicine, PO Box 7343, Wellington South, New Zealand. 